\title{
Guía de Principios Éticos de la Red Iberoamericana de Instituciones de Medicina Legal y Ciencias Forenses
}

\section{Resumen}

La Red Iberoamericana de Instituciones de Medicina Legal y Ciencias Forenses, organización que integra el Comité Internacional Cruz Roja, acordó una Guía de Principios Éticos. Sus objetivos son establecer una base mínima consensual sobre los aspectos éticos del trabajo de la Medicina Legal y las Ciencias Forenses en el contexto regional, así como ofrecer un marco axiológico de referencia para las instituciones y los profesionales forenses. Los nueve principios desarrollados en la Guía giran en torno a la obligación del respeto y promoción de los derechos humanos, la calidad técnica del trabajo, la necesaria formación en Medicina Legal y Ciencias Forenses, el trabajo en equipo respetando los perfiles disciplinares, la independencia técnica, los posibles conflictos de interés, los conflictos deontológicos cuando se actúa bajo dependencia, la confidencialidad y el consentimiento informado. Las Guías asumen también los principios consagrados en diversos documentos internacionales y en los códigos de ética nacionales. En Uruguay, el documento fue aprobado por la Facultad de Medicina y el Consejo Directivo Central de la Universidad de la República dispuso su más amplia difusión entre todos los servicios, especialmente a aquellos que participan en actividades forenses.

Palabras clave: MEDICINA LEGAL

CIENCIAS FORENSES

GUÍAS

ÉTICA MÉDICA

Key words: $\quad$ FORENSIC MEDICINE

FORENSIC SCIENCES

GUIDELINES

ETHICS, MEDICAL

\footnotetext{
Este documento fue aprobado por el Consejo de la Facultad de Medicina (Resolución № 91 de 23 de noviembre de 2016). Por disposición del Consejo Directivo Central fue ampliamente difundido entre todos los servicios universitarios, especialmente entre los que desarrollan actividades forenses (Resolución № 19 de 6 de diciembre de 2016).

Aprobado por unanimidad en el IX Encuentro de la Red Iberoamericana de Instituciones de Medicina Legal y Ciencias Forenses. La Habana, 28 de setiembre de 2016 .

Recibido: 15/2/18.

Aprobado: 20/2/18.
} 


\section{Introducción a las Guías de Principios Éticos}

Con la elaboración de estas Guías de los principios éticos y valores rectores de la Red Iberoamericana de Instituciones de Medicina Legal y Ciencias Forenses se busca contribuir a las buenas prácticas en la comunidad técnica, científica y académica.

El adecuado manejo de los aspectos éticos en el trabajo forense constituye un componente principal de la calidad y de la lex artis de las disciplinas intervinientes. Pese a ello, estos aspectos éticos del trabajo forense han tenido un desarrollo teórico relativamente escaso. Por su valor histórico y su vigencia, merecen una referencia especial los Decálogos de Edmund Locard, Nerio Rojas y Genival Veloso de França.

Por otra parte, existen instrumentos jurídicos internacionales, normas del derecho interno de los países, declaraciones, recomendaciones y códigos de ética de las asociaciones profesionales, que resultan de aplicación en el campo que nos ocupa.

Durante la VII Reunión de Managua (2014), la Red Iberoamericana de Instituciones de Medicina Legal y Ciencias Forenses acordó la necesidad de avanzar en definiciones sobre los aspectos éticos del trabajo que realizan. En la VIII Reunión en Montevideo (2015), se aprobó un documento de avance de principios éticos de la Red.

\section{Objetivos de la Guía}

1. Establecer una base mínima consensual sobre los aspectos éticos del trabajo de la Medicina Legal y las Ciencias Forenses en el contexto regional de la Red.

2. Ofrecer un marco axiológico común de referencia para las instituciones y profesionales integrantes de la Red.

\section{Normativa y documentos de referencia}

Las Instituciones de Medicina Legal y Ciencias Forenses deben cumplir su misión con apego a las normas deontológicas de las profesiones involucradas y con arreglo a los derechos humanos.

Sus principios éticos se guiarán por lo establecido en los instrumentos jurídicos internacionales que consagran los derechos humanos, los que serán considerados un estándar mínimo.

En este sentido, y sin perjuicio de otros documentos, se asumen plenamente los valores consagrados en los siguientes instrumentos jurídicos internacionales:

- La Declaración Universal de Derechos Humanos (1948).

- Convención Americana de Derechos Humanos (1969).
- El Pacto Internacional de Derechos Civiles y Políti$\cos (1976)$.

- La Convención contra la Tortura y otros Tratos o Penas Crueles, Inhumanos y Degradantes (1987).

- La Declaración Universal sobre el Genoma Humano y los Derechos Humanos (1997).

- La Declaración Internacional sobre los Datos Genéticos Humanos (2003).

- La Declaración Universal sobre Bioética y Derechos Humanos de la UNESCO (2005).

Igualmente, se asumen los criterios éticos contenidos en los siguientes documentos:

- El Protocolo de Minnesota. Manual sobre la Prevención e Investigación Eficaces de las Ejecuciones Extralegales, Arbitrarias o Sumarias, Naciones Unidas (1991).

- El Protocolo de Estambul. Manual para la investigación y documentación de la Tortura y otros Tratos o Penas Crueles, Inhumanos y Degradantes del Alto Comisionado de las Naciones Unidas para los Derechos Humanos (2000).

- La gestión de cadáveres en situación de desastre Guía práctica para equipos de respuesta, de OPS/ OMS, CICR (2010).

También se expresa la adhesión a las siguientes declaraciones, resoluciones y recomendaciones formuladas por organizaciones internacionales profesionales:

- Declaración de la Asociación Médica Mundial sobre las Investigaciones Forenses de los Desaparecidos (2013).

- Resolución de la Asociación Médica Mundial sobre la Relación entre el Derecho y la Ética (2015).

- Declaración de Tokio de la Asociación Médica Mundial. Normas Directivas para Médicos con respecto a la Tortura y otros Tratos o Castigos crueles, inhumanos o degradantes, impuestos sobre personas detenidas o encarceladas (2006).

- Declaración de la Asociación Médica Mundial sobre la Responsabilidad de los Médicos en la Documentación y la Denuncia de Casos de Tortura o Trato Cruel, Inhumano o Degradante (2007).

Finalmente, cabe señalar que son de aplicación las normas éticas nacionales vigentes en cada país -y que en algunos casos están recopilados en códigos deontológicos y tienen rango legal- para las distintas disciplinas forenses. 


\section{Principios éticos de la Red Iberoamericana de Instituciones de Medicina Legal y Ciencias Forenses}

Los siguientes son principios éticos para el desempeño de los profesionales de la Medicina Legal y Ciencias Forenses.

Esta enumeración no debe considerarse una nómina taxativa sino una enumeración de principios fundamentales en el contexto de las normas y documentos de referencia referidos.

1. Respeto y promoción de los derechos humanos. El objetivo de la Medicina Legal y las Ciencias Forenses es procurar llegar a la verdad a través de medios legales, respetando la ética profesional y los derechos humanos.

En toda actividad se observará escrupulosamente el respeto por los derechos humanos. Este criterio prioritario e inexcusable, comprende el respeto de los derechos y la dignidad de las víctimas de la violencia -incluidas sus familias y sus comunidades- así como la de los propios victimarios.

En un sentido lato, este principio incluye el respeto a la dignidad de los cadáveres recientes o esqueletizados y de las muestras biológicas de origen humano.

2. Trabajo de calidad. La actividad en el campo forense encarna una gran responsabilidad social y, además, impacta de manera directa sobre la vida de las personas involucradas en las investigaciones.

Por ello, la calidad del trabajo, el apego al método científico y la prudencia en las conclusiones pasan a tener rango de componente ético de la actuación en Medicina Legal y Ciencias Forenses.

3. Formación en Medicina Legal y Ciencias Forenses. El trabajo profesional en el campo de la Medicina Legal y Ciencias Forenses requiere una sólida formación en la disciplina madre que se ejerce, así como una formación y entrenamiento específico en el método pericial.

Tanto el intrusismo, como el adjudicarse falsamente especialización en materia forense, resultan éticamente inadmisibles.

Los miembros de la Red Iberoamericana se presentarán con la formación, experiencia y especialización pertinente, sin perjuicio de su obligación de actualizarse y desarrollar una educación continua y progresiva.

Los profesionales de la Medicina Legal y las Ciencias Forenses deberán negarse a realizar actividades periciales para las que no cuentan con la formación necesaria.

4. Trabajo en equipo. Se debe valorar y estimular el trabajo en equipo, donde cada disciplina aporte de acuerdo a su propio conocimiento específico y cada pe- rito lo haga de acuerdo a su nivel de formación y calificación.

El trabajo en equipos multi o interdisciplinarios debe ser complementario y no sustituye el rol específico de cada una de las disciplinas forenses.

5. Independencia técnica. Los profesionales de la Medicina Legal y las Ciencias Forenses deben asegurar una completa independencia técnica, rechazando y denunciando toda presión o intromisión de las autoridades, terceras personas $\mathrm{u}$ organizaciones, cualquiera fueran estas.

La empatía con las presuntas víctimas o victimarios no debe comprometer el carácter científico de las actuaciones periciales.

El perito debe ser completamente independiente en sus informes, basándose únicamente en elementos técnicos, incluso dejando de lado otras convicciones personales.

6. Conflictos de interés. En la actividad médico-legal y forense pueden presentarse diversos conflictos de interés. Muchos de ellos están detallados en los códigos procesales de cada país e inhabilitan para el desempeño pericial.

Los informes de los consultores técnicos de parte son legítimos e integran el conjunto de garantías de un proceso justo. Este tipo de informe contratado por una de las partes de un proceso será ético en tanto consigne con objetividad y rectitud científica sus conclusiones. El profesional que es contratado para un informe a solicitud de parte actúa éticamente cuando informa lo mismo que hubiera informado en caso de ser contratado por la contraparte, ofreciendo así la misma respuesta a una misma pregunta.

Es contrario a la ética actuar como perito oficial y de parte en el mismo caso, o en casos relacionados.

Los profesionales de la salud tienen una absoluta incompatibilidad ética para actuar como peritos de personas con las que tienen o tuvieron una relación clínica o terapéutica.

7. Conflictos deontológicos actuando bajo dependencia. Los profesionales de la Medicina Legal y las Ciencias Forenses pueden estar sometidos a directivas que colisionan con las reglas éticas de su profesión. En tales casos debe primar el apego a las reglas éticas, muy particularmente si esa colisión compromete los derechos humanos.

Tanto individual como colectivamente, los profesionales forenses deben reclamar a las autoridades que en el cumplimiento de su función se respeten escrupulosamente las normas éticas de su profesión. 
8. Confidencialidad. La confidencialidad es una obligación ética fundamental, toda vez que los profesionales de la Medicina Legal y las Ciencias Forenses acceden a información de la que no son titulares.

En el trabajo en el ámbito forense, donde los profesionales deben informar a las autoridades, la confidencialidad no se extingue, sino que adopta modalidades especiales. Por una parte, a la obligación del secreto profesional se añade la que corresponde al secreto de la investigación a la que están obligados los peritos. Además, la información que no sea relevante para la investigación no debería ser revelada, con especial cuidado en aquella que pueda ser causa de estigmatización o producir un daño injusto.

Durante el transcurso de la investigación, no es función de los peritos comunicar los resultados a los medios de comunicación, salvo encargo de las autoridades competentes.

Para la comunicación de casos con interés académico, son de aplicación las normas generales de la ética de la investigación y la publicación científica

9. Consentimiento informado. En Medicina Legal y Ciencias Forenses aplica la regla del consentimiento informado, aunque admite algunas excepciones y presenta ribetes singulares.

Toda persona que es objeto de interrogatorio y examen pericial tiene derecho a ser informada del propósito, los detalles de las maniobras que se efectuarán, así como de que la información recabada será puesta en conocimiento de la autoridad competente. Lo mismo aplica para la toma de muestras biológicas.

En casos de personas incapaces, los peritos se deberán regir por el derecho interno de cada país, priorizando siempre el respeto a su dignidad y sus derechos humanos. En caso de niños, niñas o adultos incapaces, el consentimiento será dado por el representante legal. Para los adolescentes aplica la doctrina de la madurez progresiva recogida en las normas internacionales y el derecho interno.

La autopsia y exhumación judicial, o la colecta de indicios biológicos en la escena del hecho, pueden no requerir consentimiento, siempre que se realicen con arreglo a la legislación nacional.

Cuando la investigación forense suponga intervenir en comunidades puede ser de recibo procurar el consentimiento comunitario.

\section{Abstract}

The Ibero American Network of Legal Medicine Institutions and Forensic Sciences, an organization that is part of the Red Cross International Committee agreed on a Guide of ethical principles with the purpose of a minimum agreed platform on ethical aspects in connection with professional practice in Legal Medicine and Forensic Sciences within the regional context. Likewise, it aims to provide an axiological framework for both forensic institutions and professionals. The nine principles agreed upon deal with the obligation to respect and promote human rights, the technical quality of work, need for training in Legal Medicine and Forensic Sciences, teamwork respecting disciplinary profiles, technical independence, potential conflicts of interest and deontological conflicts when acting under a certain rule, confidentiality and informed consent. The guide also undertakes the principles contemplated on several international documents and in the national codes of ethics. In Uruguay the document was approved by the School of Medicine and the Board of the University of the Republic decided for it to be communicated among all services, especially among those which take part in forensic activities.

\section{Resumo}

A Rede Ibero-americana de Instituições de Medicina Legal e Ciências Forenses, organização integrante do Comitê Internacional Cruz Roja, elaborou um Guia de Princípios Éticos. Seus objetivos são estabelecer uma base mínima de consenso sobre os aspectos éticos do trabalho da Medicina Legal e das Ciências Forenses no contexto regional, bem como oferecer um ponto axiológico de referencia para as instituições e profissionais forenses. Os nove princípios descritos no Guia foram desenvolvidos considerando a obrigação ao respeito e a promoção dos direitos humanos, a qualidade técnica do trabalho, a necessária formação em Medicina Legal e Ciências Forenses, o trabalho em equipe respeitando os perfis das diferentes disciplinas, a independência técnica, os possíveis conflitos de interesse, os conflitos deontológicos quando se age em condições de dependência, a confidencialidade e o consentimento informado. O Guia assume também os princípios consagrados em diversos documentos internacionais e nos códigos de ética nacionais. No Uruguai, o documento foi aprovado pela Facultad de Medicina e o Consejo Directivo Central da Universidad de la República determinou que fosse amplamente difundido entre todos os serviços e departamentos, especialmente a aqueles que participam em atividades forenses. 